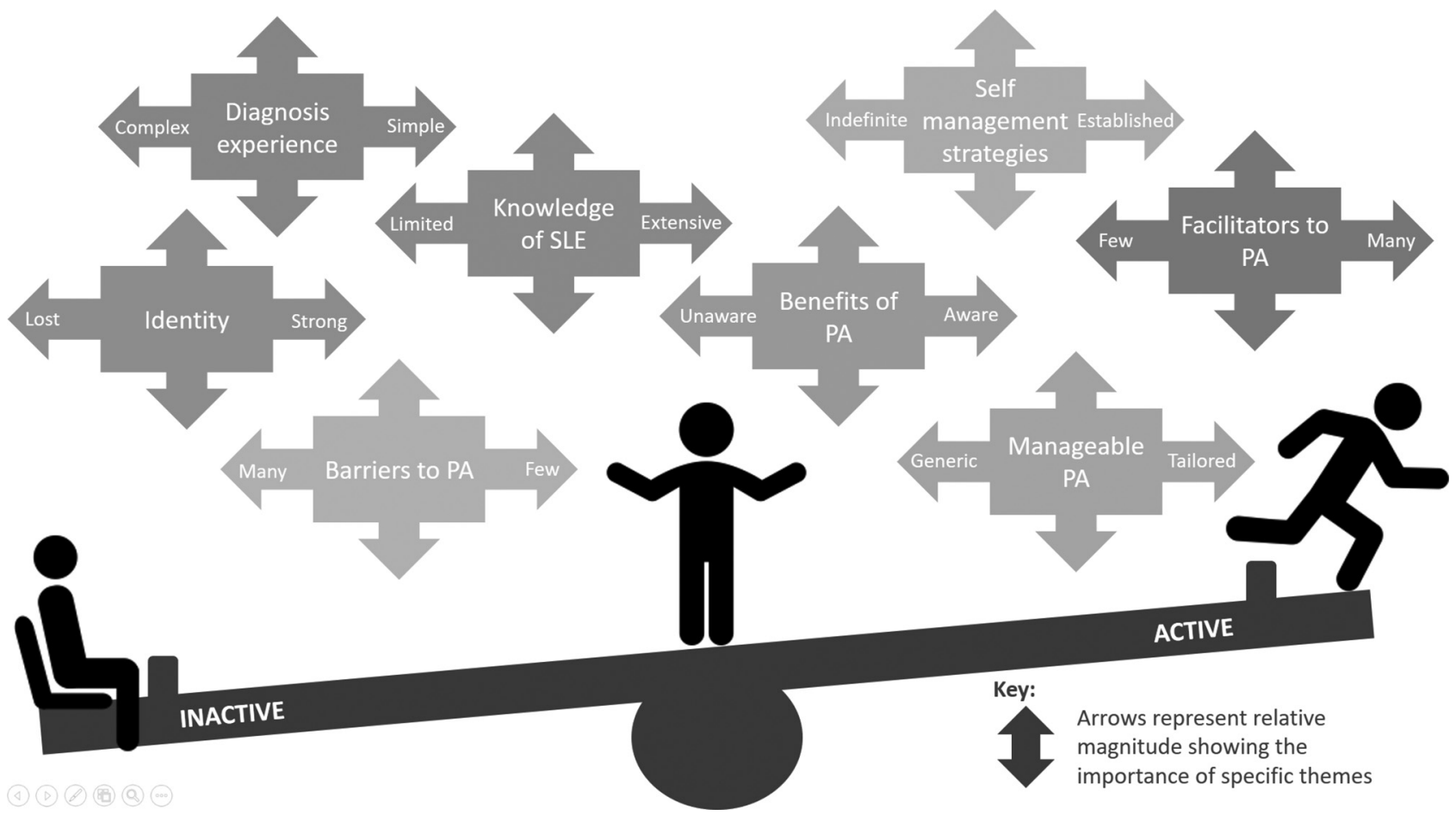

Abstract 118 Figure 2 Infographic - The PA seesaw for PwSLE. The individual with SLE is on the seesaw between being inactive and active. The themes at the inactive side may be hurdles to PA and the themes on the active side may be aids to PA. For an individual, each theme may have a more negative or positive impact on PA, moving from left to right. The importance of each theme may also vary, growing or shrinking in size, depending on the individuals circumstances. Clinicians should consider the importance and relevance of each of the themes to ensure development of tailored PA interventions for PwSLE

\section{P119 MEDICATION ADHERENCE IS INFLUENCED BY RESILIENCE IN PATIENTS WITH SYSTEMIC LUPUS ERYTHEMATOSUS}

${ }^{1,2}$ Claudia Mendoza-Pinto, 'Pamela Munguía-Realpozo, ${ }^{1}$ Sandra Campos-Rivera, ${ }^{2}$ Socorro Méndez-Martínez, ${ }^{1,2}$ Mario García-Carrasco. ${ }^{1}$ High-Specialty Medical Unit-CIBIOR, Mexican Social Security Institute, Puebla; ${ }^{2}$ Dept. of Rheumatology and Immunology, Medicine School, Meritorious Autonomous University of Puebla, Puebla, Mexico

\subsection{6/lupus-2020-eurolupus. 163}

Background/Purpose Resilience has received attention as an important process in the experience and management of chronic morbidities. ${ }^{1}$ However, few studies have evaluated resilience in patients with systemic lupus erythematosus and possible associations with treatment adherence. ${ }^{2}$ Therefore, the aim of this study was to assess the impact of resilience, the ability to withstand and bounce back from adversity, on medication adherence in SLE patients.

Methods A cross-sectional observational study was conducted in outpatients with SLE. Patients were assessed for resilience (Connor-Davison Resilience Scale, CD-RISC), depressive symptoms (Center for Epidemiologic Studies Depression Scale, CES-D) and medication adherence using the Compliance Questionnaire for Rheumatology (CQR). The disease activity index (Mex-SLEDAI) and damage index (SLICC) were administered in the patients. Factors independently associated with adherence were identified using multivariable logistic regression.

Results Of the 157 patients, 152 (96.8\%) were female with a median age of 45.9 (IQR: 39.0-55.5) years and disease duration of 14 (IQR: 10.0-19.0) years. Medication adherence (CQR $\geq 80 \%)$ and depressive symptoms were found in $74.5 \%$ and $43.9 \%$ of patients, respectively. Adherent patients had a lower CES-D score and a higher CD-RISC score. A positive correlation between resilience and adherence was found $(r=0.26, p=0.001)$. In the multivariate analysis adjusting for demographic and clinical confounders, resilience remained significantly associated with high adherence (OR 1.04 [95\% CI 1.02-1.07]). In addition, being older was also independently associated with high adherence (95\%CI 1.04 [1.0-1.07]).

Conclusions In SLE patients, resilience may be associated with better medication adherence. Therefore, a resilience-based perspective might be a new approach that focuses on enhancing strategies to improve adherence.

\section{REFERENCES}

1. Grünberg J. The impact of logistics and resilience on compliance. Pediatric Nephrol. 2005; 20;1823-1824.

2. Mehat $P$, Atiquzzaman M, Esdaile JM, et al. Medication Nonadherence in Systemic Lupus Erythematosus: A Systematic Review. Arthritis Care Res (Hoboken) 2017;69; 1706-1713.

\section{P120 POTENTIAL AND PROGNOSTIC FACTOR FOR BELIMUMAB-FREE REMISSION IN SLE PATIENTS: SINGLE-CENTER RETROSPECTIVE ANALYSIS}

Takehiro Nakai, Yukihiko Ikeda, Ayako Kitada, Masei Suda, Hiromichi Tamaki, Kenichi Yamaguchi, Masato Okada. Immuno-Rheumatology Center, Saint-Luke's International Hospital, Tokyo, Japan

\subsection{6/lupus-2020-eurolupus. 164}

Background B cells are critically involved in the pathogenesis of systemic lupus erythematosus (SLE), and their increased activity is driven in part by increased levels of growth factors, including B-lymphocyte stimulator (BLyS). Belimumab inhibits 
activity of BLyS, effectively and safely treats SLE, but data on treatment cessation are lacking. Therefore, we investigated belimumab-free remission in SLE patients.

Methods SLE patients receiving belimumab in our institute (1/ 1/2013-5/31/2019) were retrospectively identified using electronic health records. Eligibility criteria were receiving belimumab for $>180$ days and discontinuation for any reason. BILAG category A or B in at least one organ system defined disease flares. Follow-up monitoring after 52 weeks post-treatment identified relapse-free and relapse patients.

Results 31 patients received belimumab. While 14 patients discontinued, eight were included. Four patients relapsed within 52 weeks. Relapse-free patients received significantly less steroid at discontinuation (prednisolone equivalent, median 3.0 $\mathrm{mg} /$ day [IQR 2.75-3.19] vs. $9.5 \mathrm{mg} /$ day [IQR 7.25-13.25], $\mathrm{p}=0.02$ ), and significantly more of them achieved PSL dosage of $<5 \mathrm{mg} /$ day on discontinuation day than relapse patients. $(p=0.03)$ At belimumab discontinuation, relapse-free patients tended to have higher C3 (median $91.0 \mathrm{mg} / \mathrm{dL}$ [IQR 78.75102.25] vs. $56.0 \mathrm{mg} / \mathrm{dL}$ [IQR 39.75-73.00], $\mathrm{p}=0.15$ ) and $\mathrm{C} 4$ levels (median $22.0 \mathrm{mg} / \mathrm{dL}$ [IQR 19.00-26.00] vs. $11.0 \mathrm{mg} / \mathrm{dL}$ [IQR 6.00-16.00], $\mathrm{p}=0.08$ ) and less anti-DNA antibody (median 5.2 IU/mL [IQR 3.75-7.83] vs. $48.0 \mathrm{IU} / \mathrm{mL}[\mathrm{IQR}$ 11.50-137.25I], $\mathrm{p}=0.08$ ) than relapse patients, but differences were not significant.

Conclusion Belimumab discontinuation after $>180$ days is recommended for $50 \%$ of SLE patients. Steroid dosage (prednisolone equivalent $<5 \mathrm{mg} /$ day) might be a prognostic marker for belimumab-free remission.

Funding The authors received no financial support for the research, authorship, and/or publication of this article.

\section{P121 IDENTIFICATION AND ACTIVE MANAGEMENT OF SECONDARY ADRENAL INSUFFICIENCY IN SLE REDUCES RISK OF RELAPSE}

${ }^{1}$ Benjamin Rhodes, ${ }^{2}$ Haroon Ahmad, ${ }^{3}$ Sarah Logan, ${ }^{1}$ Elizabeth Rankin, ${ }^{3}$ Peter Hewins. ${ }^{1}$ Rheumatology Dept., Queen Elizabeth Hospital, Birmingham; ${ }^{2}$ University of Birmingham Medical School, Birmingham; ${ }^{3}$ Nephrology Dept., Queen Elizabeth Hospital, Birmingham, UK

10.1136/lupus-2020-eurolupus. 165

Background Steroids are an important component of Lupus treatment, but long-term use is associated with an accumulation of damage, emphasising the importance of optimising steroid withdrawal protocols. The factors influencing successful steroid withdrawal in SLE are poorly understood. Secondary adrenal insufficiency is common following therapeutic steroid usage, but the prevalence in steroid-treated lupus patients is undocumented. We documented rates of adrenal insufficiency and evaluated whether actively managing steroid withdrawal influenced the risk of disease relapse.

Methods 137 SLE patients who had been on Prednisolone for more than a year before reducing the dose below $5 \mathrm{mg} /$ day for the first time were retrospectively reviewed, documenting the clinician decision to screening for adrenal insufficiency, the frequency of adrenal insufficiency, the method of steroid reduction (stopping vs. tapering regimens), clinical, pharmacological and immunological variables. Parameters influencing the risk of relapse from the point of dropping below $5 \mathrm{mg} /$ day of Prednisolone were evaluated by Cox' regression.
Results 65 patients were screened for adrenal insufficiency. $38 \%$ failed initial screening and $12 \%$ showed no signs of adrenal recovery after more than a year. $46.7 \%$ returned to higher dose steroids, with $34 \%$ having a true disease relapse (BILAG A or B flare). In a multivariate model the decision to screen for adrenal insufficiency was the variable most strongly associated with risk of disease relapse (BILAG A/B flare) (marginal Hazard Ratio 4.33, $\mathrm{P}=0.0001$ ), followed by complement C4 at baseline and South Asian ancestry. Concomitant medications or the method of steroid reduction (stopping vs. tapering) did not influence relapse. A full analysis of factors associated with failed adrenal screening and risk of disease relapse will be presented.

Conclusions Adrenal insufficiency in common in patients with SLE and unless screening takes place this may go undetected. Detection and active management of adrenal insufficiency is associated with a significant and meaningful reduction in disease relapse.

\section{P122 WHAT DO PATIENTS WITH LUPUS AND SJÖGREN'S SYNDROME KNOW ABOUT CARDIOVASCULAR RISK?}

Elvira Chocano Navarro, George Robinson, Kirsty Waddington, Thomas McDonnell, Chris Wincup, Lucia Martin-Gutierrez, Annalisa Maggio, Eve McLoughlin, Lizzy Rosser, David Isenberg, Anastasia Kalea, Coziana Ciurtin, Ines Pineda-Torra, Elizabeth Jury. Dept. of Medicine, University College London, London, UK

\subsection{6/lupus-2020-eurolupus.166}

Background Cardiovascular risk (CVR) is the leading cause of mortality in patients with lupus. Understanding increased CVR in autoimmune rheumatic diseases could improve management of risk in patients. This event aimed to explore patient opinions about CVR and potential CVR treatment options.

Methods We hosted a patient event promoted through social media, relevant charities, hospitals and research groups. 13 patients with lupus and/or Sjögren's syndrome attended and were asked about CVR using a questionnaire (14 questions) and round table discussion with patients, researchers, clinicians and dietitians.

Results Sixty percent of patients were aware of the increased CVR associated with autoimmune rheumatic disease and $60 \%$ stated that their doctor had spoken to them about this risk. $73 \%$ thought that it was important for them to be aware of this increased CVR.

When asked about medication to reduce CVR, no patients wanted to take a statin (lipid lowering drug), however, $70 \%$ of patients would take statins if advised to do so by their doctor. Conversely, respondents were more positive about using diet or taking a dietary supplement to reduce CVR; $71 \%$ would change their diet and $57 \%$ would take a supplement either on their own accord or on advice from health professionals. Some patients had already made changes to their diet to reduce their CVR, including reducing fat and increasing fruit and vegetable consumption. All attendees were prepared to participate in a clinical study using diet modification strategies, having vascular scans to assess atherosclerosis and provide blood samples for CVR research in lupus/Sjögren's syndrome.

Conclusion This multidisciplinary event successfully gathered patient information regarding CVR. The opinions and comments provided evidence that patients support further research in cardiovascular studies and a preference to changing their diet or take a supplement, whilst avoiding medication, to reduce their CVR. 